\title{
NOUVELLES COCCIDIES CHEZ LES GERBOISES
}

\section{(ALACTAGA JACULUS)}

\author{
Par W. L. YAKIMOFF et W. F. GOUSSEFF
}

En 1935, Mme Iwanoff-Gobzem trouva, en Kazakstan du Nord, chez une gerboise (Alactaga jaculus Pall.) une coccidie. Les oocystes ont la forme ronde, avec une enveloppe mince et à double contour. Le protoplasme granuleux occupe le quart de l'oocyste. L'oocyste non sporulé contient un granule polaire. Les dimensions des oocystes sont : 22,3-26,4 $\mu$, en moyenne 23,3 $\mu$. Après culture, on obtient dans l'oocyste quatre spores. $\mathrm{Ni}$ l'oocyste, ni les sporocystes n'ont de reliquats. L'auteur a nommé cette coccidie Eimeria alactagx.

Nous avons trouvé dans la contrée Azow-Mer Noire (rayon du Nord du Don, près la station Baski), chez une gerboise (Alactaga jaculus), deux coccidies du genre Eimeria.

Première espèce :

La forme des oocystes est ronde, leur couleur jaune-orange. Sans micropile. Dimensions : $16 \mu, 7-18 \mu, 4$, en moyenne $17 \mu, 4$, plus souvent $16,7 \mu$.

Dans une solution de bichromate de potasse se développèrent quatre spores $(9 \mu, 2 \times 7 \mu, 7)$, de forme largement ovale, avec deux sporozoïtes, chacun en forme de virgule.

Après avoir sporulé, ce ne sont que les sporocystes qui présentent des reliquats; l'oocyste n'en a pas. Point de granule polaire dans les oocystes, ni avant, ni après avoir sporulé.

Notre coccidie ne ressemble, ni en dimensions, ni par l'absence d'un granule polaire dans l'oocyste, à la coccidie trouvée par Mme Iwanoff-Gobzem.

Nous considérons cette coccidie comme une nouvelle espèce et nous la nommons, en l'honneur du savant français, le professeur G. Lavier, Eimeria lavieri n. sp.

\section{DEUXIÈME ESPÈCE :}

La forme de l'oocyste est subsphérique. La couleur est jaune. Dimensions : $34 \mu, 5-27 \mu, 5 \times 21 \mu, 4$, en moyenne, $26 \mu, 4 \times 21 \mu, 4$,

Annales de Parasitologie, $\mathrm{T}$. XIV, $\mathrm{N}^{\circ} 5 .-1^{\mathrm{er}}$ septembre 1936. 
maximum $27 \mu, 5 \times 21 \mu, 4$, minimum $24, \mu, 5 \times 21 \mu, 5$, plus souvent $27 \mu, 5 \times 21 \mu, 4$. Formindice : $1: 0,78-0,87$, en moyenne $1: 0,81$, plus souvent $1: 0,78$.

Dans la solution de bichromate de potasse, se développent dans l'oocyste quatre spores $(13 \mu, 6 \times 10 \mu, 6)$, de forme largement

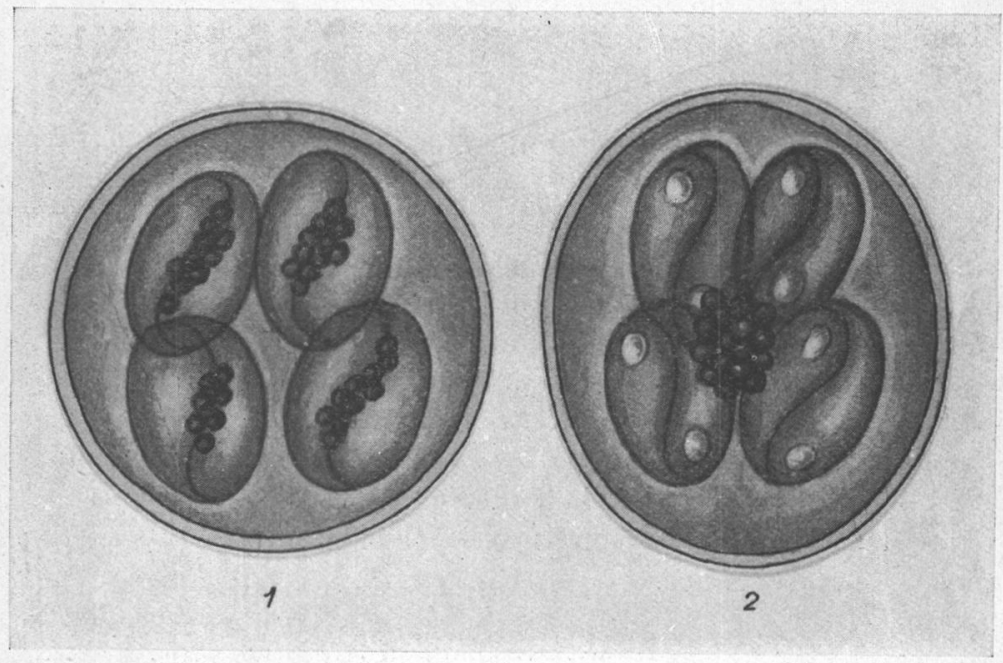

Fig. - 1, Eimeria lavieri n. sp. ; 2, Eimeria joyeuxi n. sp.

ovale, avec deux sporozoïtes en forme de virgule dans chacune d'elles.

Après la sporulation, l'oocyste montre un reliquat qui manque dans les sporocystes. Point de granule polaire dans l'oocyste.

Nous considérons cette coccidie comme nouvelle et nous la nommons, en l'honneur du savant français, le professeur Ch. Joyeux, Eimeria joyeuxi n. sp.

\section{BibLIOGRAPHIE}

IWANOFF-Gobzem (P.-S.). - Zum Vorkommen von Coceidien bei kleinen wilden Säugetieren. Deutsche tier. Wochenschr., 1934, p. 149-151.

Laboratoire de Parasitologie de l'Ecole vétérinaire de Léningrade et Section de Protozoologie de l'Institut vétérinaire scientifique de Nowotscherkassk. 